\title{
ARTICLE
}

\section{New diagnostic concepts in Alzheimer's disease}

\author{
Anna Watkin, Sudip Sikdar, Biswadeep Majumdar \& Anna V. Richman
}

\begin{abstract}
Anna Watkin is an ST4 trainee in Mersey Deanery Old Age Psychiatry rotation. Sudip Sikdar is a clinical director and consultant in old age psychiatry in the Mersey Care NHS Trust and a regional advisor in psychiatry in Mersey. Biswadeep Majumdar and Anna Richman are consultants in old age psychiatry in Mersey Care NHS Trust. Correspondence Dr Sudip Sikdar, Waterloo Day Hospital, Park Road, Waterloo, Liverpool L22 3XR, UK. Email: Sudip.Sikdar@merseycare. nhs.uk
\end{abstract}

a. NINCDS-ADRDA, the National Institute of Neurological and Communicative Disorders and Stroke and the Alzheimer's Disease and Related Disorders Association (the latter is now known as the Alzheimer's Association)

\begin{abstract}
SUMMARY
This article gives an overview of the profile of Alzheimer's disease, its pathophysiology and recent developments in technology that enable better understanding of the mechanism of disease. The diagnostic criteria and role of biomarkers proposed are explained. The new subgroups described are outlined in table form for easy reference. Subtypes of mild cognitive impairment ( $\mathrm{MCl}$ ) are reviewed and the conversion of amnestic $\mathrm{MCl}$ to Alzheimer's disease is considered. The implications and change to current clinical practice form the basis of the conclusion of the article.
\end{abstract}

\section{DECLARATION OF INTEREST}

None.

Alzheimer's disease accounts for around 60\% of all dementias. The criteria for diagnosis of Alzheimer's disease used in clinical guidelines on dementia care within the UK's National Health Service (National Institute for Health and Clinical Excellence 2012) are based on those proposed in 1984 by the NINCDS-ADRDA ${ }^{\text {a }}$ workgroup (McKhann 1984). Our understanding of Alzheimer's disease has since evolved. Recent research into early stages of the disease process has led to a need to redefine the diagnostic criteria and recognise the stages leading up to dementia. The updated criteria developed by international workgroups convened by the National Institute on Aging and the Alzheimer's Association (the NIAAlzheimer's Association workgroups) describe three different clinical groups (Jack 2011):

- dementia due to Alzheimer's disease

- mild cognitive impairment (MCI) due to Alzheimer's disease

- preclinical Alzheimer's disease.

Table 1 shows some of the terms that have been used over the years to describe similar concepts.

\section{A reason for change}

The 1984 NINCDS-ADRDA criteria were based on a clinicopathological concept: the presence of neuropathological changes coexisting with the clinical manifestation of cognitive decline which would have an impact on social and occupational functioning. Put simply, disease of the tissue directly causes the symptoms. Neuropathological investigation is not practical in live populations, so clinical history and neuropsychological testing have dominated the criteria for diagnosis. This gave scope for variation in clinicians' ability to diagnose correctly with a certain degree of confidence. The 1984 criteria promoted 'probable' and 'possible' diagnosis to be used clinically - only autopsy confirmation would lead to a 'definite' diagnosis (McKhann 2011).

Emerging research evidence would suggest that the classic clinicopathological entity, where the presence of neuropathology and symptoms are simultaneous, may not hold true. Clinicopathological studies have shown that in about one in three cases where autopsy revealed Alzheimer's disease pathology, the individuals had not shown dementia when alive (Jack 2002). Jack et al conjectured that, had these individuals lived longer, they may have developed Alzheimer's disease. This suggests that neuropathological changes can occur years before symptoms are detected. Scientific advances over the past 20 years have offered possibilities of in vivo testing that have improved our understanding of Alzheimer's disease pathology and can provide evidence to support clinicians' diagnoses.

\section{The pathology of Alzheimer's disease}

The current generally accepted model of the disease is one of gradual accumulation of amyloid $\beta(\mathrm{A} \beta)$ in the brain, leading to synaptic dysfunction, tangle formation and neuronal death, thus contributing to cognitive decline (Sperling 2011). A number of factors, such as cerebrovascular risk, other agerelated brain disease and genetics, may contribute to the development of clinical Alzheimer's disease.

Three causative genes have been associated with autosomal dominant familial Alzheimer's disease (APP, PSEN1 and PSEN2) and one genetic risk factor (APOEe4) has been identified for late-onset sporadic Alzheimer's disease. Polymorphisms in different susceptibility genes on chromosome 12 (A2M, LRP1, CP2 and OLR1) are now being 
suggested as possible genetic markers for increased risk of developing Alzheimer's disease (Alagiakrishnan 2012).

\section{Neuroprotective mechanisms}

In an attempt to understand variations within this population, protective hypotheses such as brain and cognitive reserve have been proposed. 'Brain reserve' is conceptualised as the brain's capacity for resilience when pathological insult occurs. 'Cognitive reserve' refers to the brain's use of alternative approaches when adapting to pathology (Sperling 2011).

The concept of neuroprotective mechanisms has appeal to the general public, and a new industry of brain training and 'use it or lose it' has arisen (Doraiswamy 2012). A large UK-based population study exploring the links between an active cognitive lifestyle and reduced risk of dementia (Valenzuela 2012) suggests that, although cognitive activity benefits all, the mechanism of neuroprotection varies according to gender. Among those who had had a high lifespan Cognitive Lifestyle Score, autopsy suggested that modification of risk factors linked to cerebrovascular disease is protective for men, whereas greater brain weight may protect women. The team suggested that even when vascular risk factors are adjusted for in men, those with active cognitive lifestyles had lower rates of dementia at death. Thus, the neuroprotective effects of an active cognitive lifestyle may have multiple biological mechanisms.

A combination of genetic risk factors, other disease processes and resilience of the brain could explain the variation in the lag period between the onset of the pathological process of amyloid- $\beta$ build up and clinical Alzheimer's disease - the pathophysiological cascade model (Fig. 1). The lag could also account for autopsy results that detect pathology in 'normal' individuals who may have developed the disease had they lived long enough (Sperling 2011).

\section{Preclinical Alzheimer's disease}

Sperling et al (2011) use the amyloid- $\beta$ accumulation model to redefine the earliest stages of Alzheimer's disease years before clinical changes occur by using biomarker changes to identify this population. They argue that treatment should start before significant cognitive impairment is evident, describing this as the 'preclinical' stage. Drawing parallels with cardiac disease and cancer, they hypothesise that the use of prevention strategies would lead to greater socioeconomic improvement.

Another patient group that could be included in the preclinical domain are those who show

\section{TABLE 1 Definition of terms in Alzheimer's disease}

\begin{tabular}{|c|c|}
\hline Preclinical Alzheimer's disease & $\begin{array}{l}\text { No cognitive symptoms } \\
\text { Changes in biomarkers or challenging neuropsychological } \\
\text { testing suggestive of Alzheimer's pathology }\end{array}$ \\
\hline Pre-mild cognitive impairment & $\begin{array}{l}\text { Subtle changes in personality and cognitive abilities not } \\
\text { detected in neuropsychological testing }\end{array}$ \\
\hline Mild cognitive impairment (MCl) & $\begin{array}{l}\text { Cognitive symptoms, impairment of no lower than } \\
1.5 \text { standard deviations in cognitive assessments and } \\
\text { independent in functional abilities }\end{array}$ \\
\hline Amnestic MCl & $\begin{array}{l}\text { As } \mathrm{MCl} \text {, with poor performance on delayed recall and } \\
\text { executive function tests } \\
\text { Individuals in this category have higher risk of progression } \\
\text { to Alzheimer's disease, but can also include those with } \\
\text { vascular dementia and depression }\end{array}$ \\
\hline Prodromal Alzheimer's disease & $\begin{array}{l}\text { As } \mathrm{MCl} \text {, with neuropsychological profile specific to } \\
\text { hippocampal amnestic syndrome and biomarkers } \\
\text { suggestive of Alzheimer's disease }\end{array}$ \\
\hline Non-amnestic $\mathrm{MCl}$ & $\begin{array}{l}\text { As } \mathrm{MCl} \text {, with more prominent executive dysfunction } \\
\text { Associated with progression to frontotemporal dementia, } \\
\text { dementia with Lewy bodies and vascular dementia }\end{array}$ \\
\hline $\begin{array}{l}\text { Cognitive impairment, no } \\
\text { dementia (CIND) }\end{array}$ & $\begin{array}{l}\text { Cognitive impairment due to a variety of disorders } \\
\text { Used in research to classify the group between healthy } \\
\text { population and dementia }\end{array}$ \\
\hline $\begin{array}{l}\text { Vascular cognitive impairment } \\
\text { (VCI) }\end{array}$ & $\begin{array}{l}\text { Cognitive impairment secondary to vascular causes } \\
\text { Definition does not specify whether there is presence or } \\
\text { absence of functional impairment }\end{array}$ \\
\hline $\begin{array}{l}\text { Vascular cognitive impairment, } \\
\text { no dementia (VCIND) }\end{array}$ & $\begin{array}{l}\text { The subgroup within } \mathrm{VCl} \text { with functioning at the level of } \\
\mathrm{MCl}\end{array}$ \\
\hline $\begin{array}{l}\text { Dementia due to Alzheimer's } \\
\text { disease }\end{array}$ & $\begin{array}{l}\text { Cognitive symptoms detected, with functional impairment } \\
\text { and characteristics of Alzheimer's disease }\end{array}$ \\
\hline
\end{tabular}

no identifiable deficits on neuropsychological testing, but have subtle changes in personality and cognitive abilities. Duara et al (2011) suggested the term 'pre-MCI' for this group.

\section{Biomarkers}

The biomarker model (Table $2 \&$ Fig. 2) indicates that the first change evident at the preclinical stage involves $\beta$ amyloidosis. This key early event results in amyloid- $\beta$ peptide production which eventually leads to plaque formation. Amyloid $\beta$ is not a

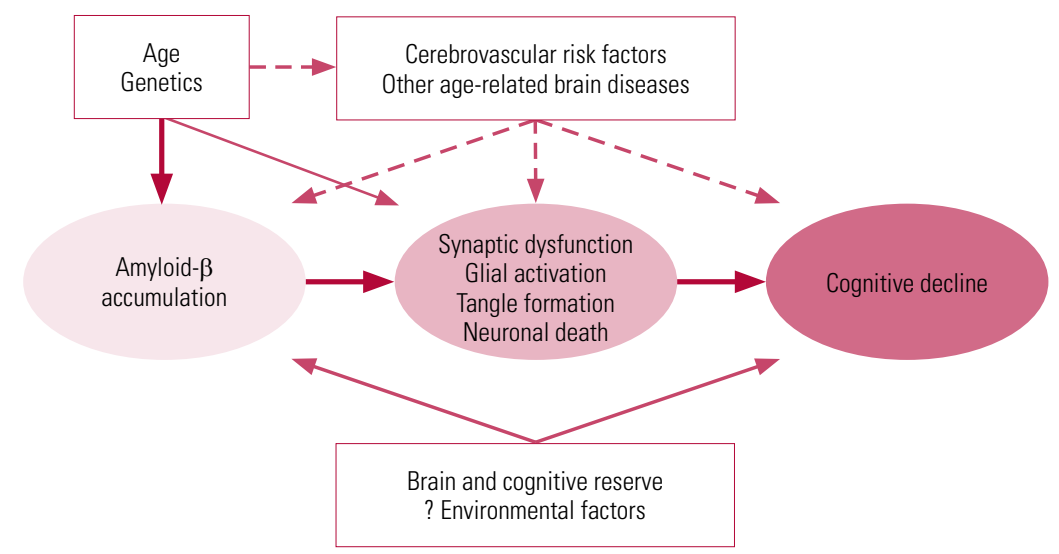

Hypothetical model of the pathophysiological cascade in Alzheimer's disease (Sperling 2011, () 2011 Elsevier, reprinted with permission). 
TABLE 2 Markers of Alzheimer's disease and predictive windows before clinical symptoms appear

\begin{tabular}{|c|c|c|c|}
\hline Marker & Predictive window & Test & Pathology \\
\hline \multirow[t]{3}{*}{ Amyloid $\beta 42$} & 25 years & CSF & Plaque formation \\
\hline & 15 years & Blood plasma & \\
\hline & 15 years & PET scan & \\
\hline $\begin{array}{l}\text { FDG } \\
\text { Tau proteins }\end{array}$ & 15 years & $\begin{array}{l}\text { PET } \\
\text { CSF }\end{array}$ & $\begin{array}{l}\text { Temporoparietal } \\
\text { hypometabolism } \\
\text { Synaptic dysfunction } \\
\text { Neuronal injury }\end{array}$ \\
\hline Hippocampal volume & 15 years & MRI & Brain atrophy \\
\hline $\begin{array}{l}\text { F18 florbetapir } \\
\text { F18 flutemetamol } \\
\text { F18 florbetaben }\end{array}$ & 10 years & PET scan & $\beta$ amyloidosis \\
\hline $\begin{array}{l}\text { Bedside neuro- } \\
\text { cognitive testing }\end{array}$ & 5 years & & Cognitive symptoms \\
\hline
\end{tabular}

CSF, cerebrospinal fluid; FDG, ${ }^{18} \mathrm{~F}$-fluorodeoxyglucose; MRI, magnetic resonance imaging; PET, positron emission tomography.

single molecular entity. It can exist as monomers, dimers, oligomers, protofibrils, fibrils and fibrillar aggregates (Walsh 2007). The amyloid $\beta 42$ (A $\beta 42)$ variant, which makes up less than $10 \%$ of total amyloid $\beta$, is more prone to aggregate than the more prominent $\beta 40$ variant. Levels of $A \beta 42$ can be measured in serum and cerebrospinal fluid (CSF). Production of amyloid $\beta$ is also thought to be fairly specific to the disease process of Alzheimer's disease. Proposed mechanisms for amyloid$\beta$-mediated 'neurotoxicity' include structural damage to the synapse, oxidative stress, altered calcium homeostasis, induction of apoptosis, structural damage, chronic inflammation and neuronal formation of amyloid pores. The reduced CSF level of A $\beta 42$ in Alzheimer's disease is believed to be caused by deposition of $A \beta 42$ in

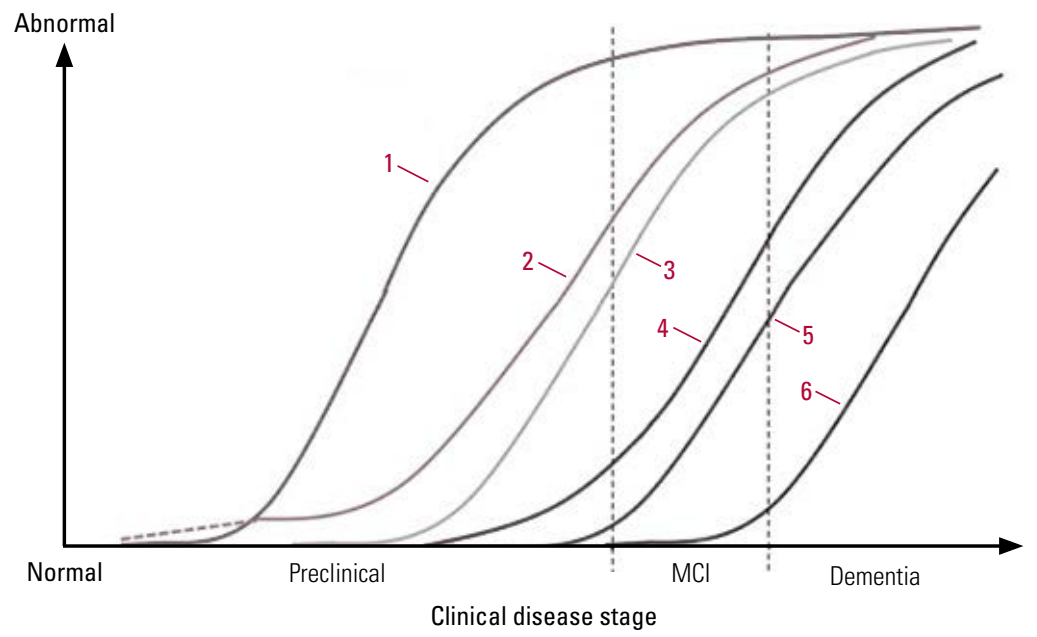

Model of dynamic biomarkers of the Alzheimer's pathological cascade. 1, amyloid- $\beta$ accumulation (CSF/PET); 2, synaptic dysfunction (FDG-PET/MRI); 3, tau-mediated neuronal injury (CSF); 4, brain structure (volumetirc MRI); 5, cognition; 6, clinical function; $\mathrm{MCl}$, mild cognitive impairment (Jack 2010, (C) 2010 Elsevier, reprinted with permission). plaques, with lower levels diffusing into the CSF. Studies have found strong correlation between low $\mathrm{A} \beta 42$ in the CSF and high numbers of plaques in the neocortex and hippocampus (Strozyk 2003) and positron emission tomography (PET) scans of the living brain that reveal plaque pathology (Fagan 2006; Forsberg 2008).

The biomarker model stipulates that the amyloid- $\beta$ accumulation is necessary but not sufficient to lead to the clinical phase of Alzheimer's disease, thereby explaining individual variations in the lag phase (Sperling 2011). Bateman et al (2012) suggest that CSF levels of A $\beta 42$ can be reduced 25 years before the estimated onset of symptoms in dominantly inherited Alzheimer's disease. They estimated that PET imaging can predict changes 15 years before symptoms occur. This is in contrast to the predictive power of episodic memory loss detected by bedside neurocognitive testing 10 years prior to clinical diagnosis, and Clinical Dementia Rating scale (CDR) changes noted 5 years prior to diagnosis.

The biomarkers involved in synaptic dysfunction are decreased ${ }^{18} \mathrm{~F}$-fluorodeoxyglucose (FDG) uptake on PET and functional magnetic resonance imaging (fMRI) abnormalities. At this point clinical manifestations such as MCI occur which may progress to Alzheimer's disease. Structural MRI changes occur as a result of neuronal loss. It has been proposed that levels of tau proteins in the CSF are related to neuronal injury, but not specifically to Alzheimer's disease (Sperling 2011).

\section{The evidence base}

The evidence base for the biomarker model is still in its infancy and the preclinical stage for Alzheimer's disease is currently used only in the research domain. Individuals at this stage would show changes in the biomarkers, but have no symptoms of cognitive decline. The biomarkers themselves are described as 'proxies' and provide only a very limited understanding of the biological process occurring in the brain (Sperling 2011). Further research is needed to establish and validate the thresholds for the biomarkers.

\section{Mild cognitive impairment - development of the term}

In clinical practice, there are inherent difficulties in differentiating 'normal' individuals from those who do not fulfil the diagnostic criteria of dementia but have some suggestive features and those with clear disease. Consequently, a spectrum of disease has been proposed on which a diagnosis of mild cognitive impairment (MCI) has been universally 
adopted. Mild cognitive impairment exists where there are cognitive changes but no functional or skills loss (Gauthier 2006).

Historically, MCI has had many names, each emphasising one factor over another or focused on specific diagnostic criteria. In 1858, Kral proposed two types of memory disturbance: benign senescent forgetfulness and malignant senescent forgetfulness. He used neuropsychiatric examination, psychological testing, and personal and social functioning to classify individuals in a nursing home. Malignant senescent forgetfulness was considered to be part of a syndrome that led to psychosis with senile brain disease, whereas benign senescent forgetfulness was not considered pathological, rather it was a subclassification of normal ageing. It has been suggested that benign senescent forgetfulness was the earliest label of MCI (Heinik 2010).

In the 1980s, Crook and colleagues proposed the diagnosis of age-associated memory impairment (AAMI) for individuals over the age of 50 who scored 1 standard deviation below young healthy controls and were otherwise unimpaired in cognitive function. They stressed that the memory impairment in AAMI is not caused by neurological, psychiatric or medical conditions and, as evidenced by their specification of using young controls, implicate ageing as the causal mechanism. The construct of AAMI has been criticised because comparison with young norms may lead to over-inclusion of neurologically normal individuals (Feldman 2005).

Age-associated cognitive decline (AACD), a term coined by Levi in the 1990s, broadened the domains included for cognitive decline into realms of learning, memory, attention, thinking, language and visuospatial function (Feldman 2005).

Cognitive impairment may result from multiple pathologies and to reflect this the term cognitive impairment, no dementia (CIND) was introduced (Plassman 2008). The term has been used in population-based studies to classify individuals whose cognitive functions lay between those of healthy people and people with dementia, and it includes a variety of disorders such as chronic alcohol or drug use, psychiatric illness and vascular disease (Feldman 2005).

In the 1990s, Petersen's use of the term mild cognitive impairment initially focused on isolated deficits in memory, but it was extended to include a range of cognitive symptoms, thus widening its scope. The broad category of MCI has been subcategorised into amnestic and non-amnestic varieties. Impairment occurring in a single cognitive domain as opposed to multiple domains can aid clinicians in distinguishing aetiology, as can be seen in Fig. 3 (Gauthier 2006).

\section{Current understanding of $\mathrm{MCI}$}

By establishing aetiology one generally can predict progression of MCI towards dementing illness. The overall rate of conversion from MCI to Alzheimer's disease has been estimated at between 6 and 25\% of cases per year (Petersen 2001), but the amnestic subtype has a conversion rate of nearly $30 \%$. The variation in the rates could be explained by the differing disease processes. In Alzheimer's disease, the progression and course of the disease led Dubois $\&$ Albert (2004) to label amnestic MCI a precursor of Alzheimer's disease. They later used the term prodromal Alzheimer's disease for those who had episodic memory loss but without functional impairment and showed changes in biomarkers supportive of Alzheimer's disease (Dubois 2010).

\section{Vascular cognitive impairment}

Conversely, vascular disease leading to vascular dementia can be variable and therefore MCI with vascular causes may take a different course. Cognitive impairment linked to vascular disease has been called vascular cognitive impairment (VCI). This term is intended for all levels of cognitive impairment linked to vascular disease, including vascular dementia. Vascular cognitive impairment, no dementia (VCIND) has been used to describe the MCI subgroup within VCI (Stephan 2009).

\section{Clinical diagnosis of $\mathrm{MCl}$}

Clinical diagnosis of MCI would generally be arrived at using information gathered from the patient or carers and results of neuropsychological diagnostic tools or clinic-based neuropsychological testing. The history would show impairment in one or more cognitive domains but no overall

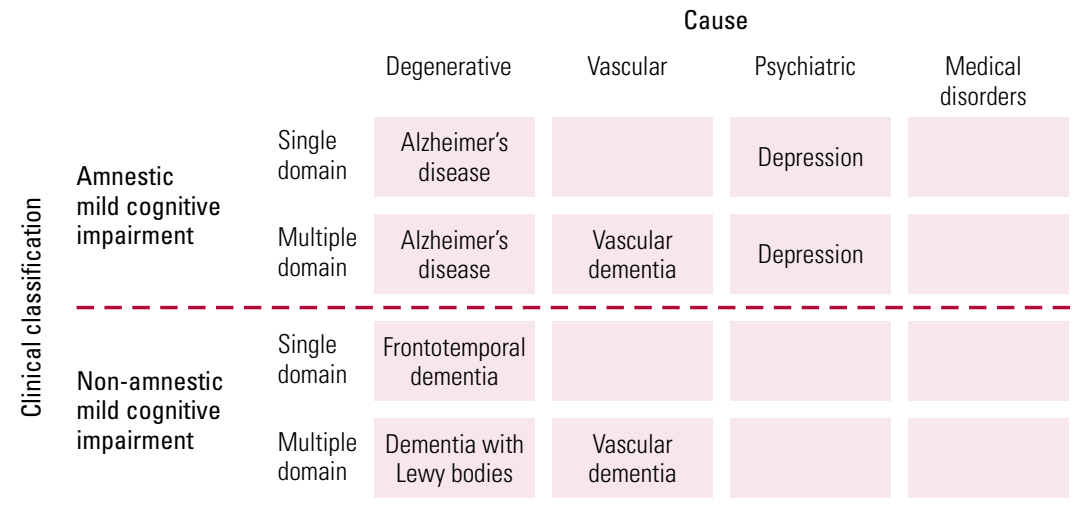

Outline of the syndrome of mild cognitive impairment (Gauthier 2006, (c) 2010 Elsevier, reprinted with permission). 
impairment in functional abilities. The cognitive assessment scores should typically be no lower than 1.5 standard deviations below the mean, matched to age and educational level (Albert 2011).

There are no fixed transition points between MCI and Alzheimer's disease, and often the functional impairment defines the diagnostic group to which individuals are allocated. Clinically, assessment of functional impairment is derived from information gathered from a carer or partner, who often dismisses 'small things' in an effort to normalise. Epidemiological studies have noted subtle difficulties in complex activities in individuals diagnosed with MCI 2 years before a diagnosis of dementia (Bateman 2012). It is therefore possible that clinicians are over-diagnosing MCI in individuals who should theoretically have a diagnosis of dementia. Formal functional testing of groups of patients with current diagnosis of MCI would probably reveal functional impairments in some and therefore their diagnoses would change to dementia.

\section{The new diagnostic criteria for Alzheimer's disease}

The NIA-Alzheimer's Association workgroups were tasked with revising the diagnostic criteria of Alzheimer's disease to encompass the developments described above. They proposed a tiered system which establishes the level of impairment and thereby delineates whether the patient has dementia or MCI or normal cognitive functions for their age (Fig. 4). Once the criteria for all-cause dementia are reached, the case is considered regarding the criteria for Alzheimer's disease (McKhann 2011).

There are other disorders that present in a similar fashion to Alzheimer's disease. Disorders such as dementia with Lewy bodies, vascular dementia and behavioural frontotemporal dementia were not fully understood and therefore not acknowledged in the 1984 NINCDS-ADRDA criteria. Indeed, the understanding of Alzheimer's disease that does not present with memory impairment as the primary complaint has since developed and needed to be incorporated into the diagnostic framework.

\section{Using the new criteria}

Criteria for all-cause dementia can be established from history (from the patient and/or carer), observation and neuropsychological testing. There should be a decline from the previous level of cognition and impairment in function which cannot be explained by delirium or major psychiatric disorder. The cognitive impairment

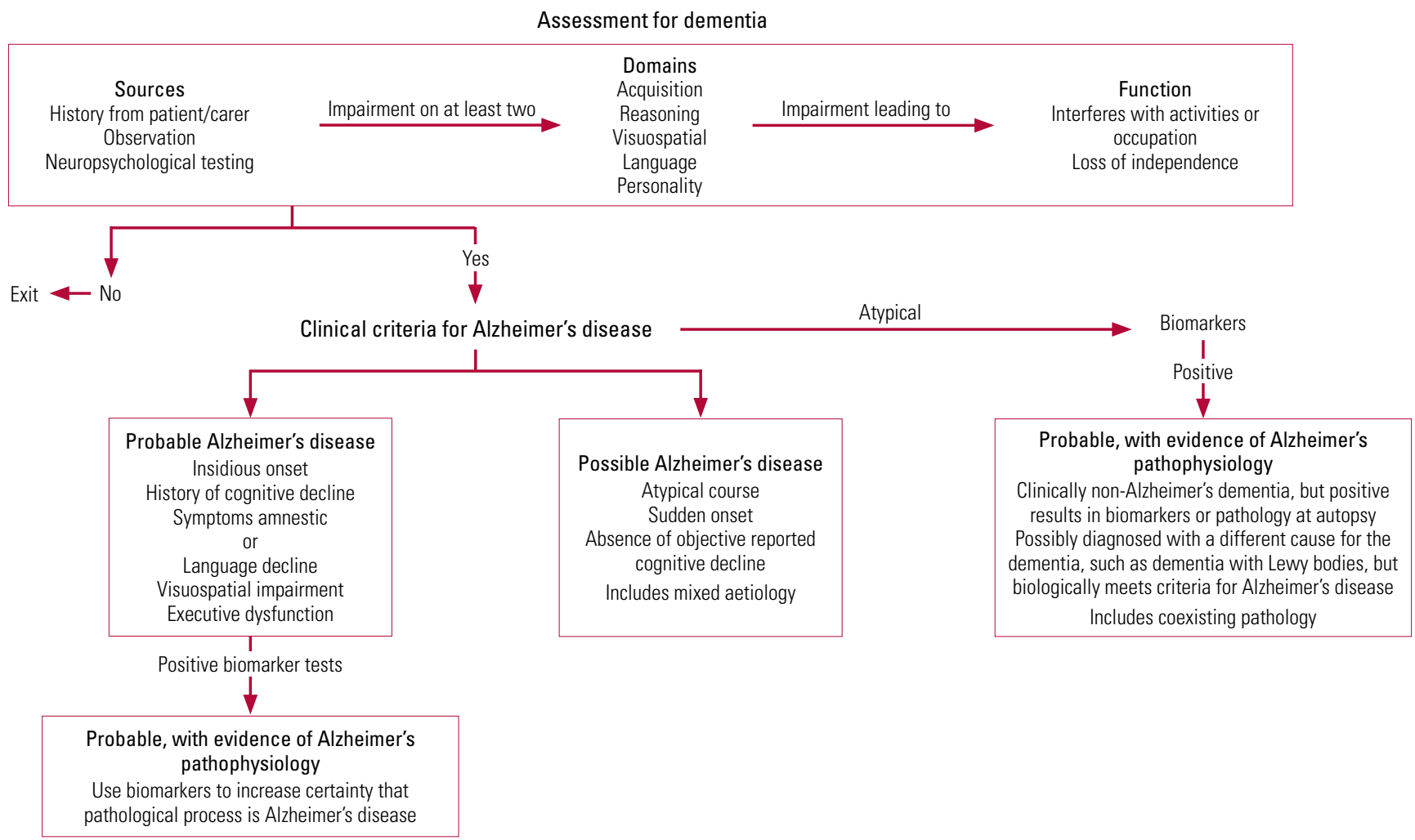


should involve a minimum of two domains from acquisition, reasoning, visuospatial, language and personality. The impairment in function should cause interference with activities or occupation (McKhann 2011).

Should the core criteria be met for all-cause dementia, the case should then be considered for the criteria for Alzheimer's disease. McKhann et al proposed three criteria sets, which result in the diagnoses of 'probable Alzheimer's disease', 'possible Alzheimer's disease' and 'probable or possible Alzheimer's dementia with evidence of the Alzheimer's pathophysiological process'.

\section{Probable Alzheimer's disease}

The criteria for probable Alzheimer's disease include insidious onset and history of cognitive decline by report or observation. The most prominent symptoms are amnestic in nature, or in the non-amnestic domains of language, visuospatial or executive dysfunction. Exclusion criteria include a history of concomitant disease such as cerebrovascular disease and presentations that would be in keeping with other forms of dementia. The certainty of the diagnosis increases with evidence of either documented decline in cognitive functions on subsequent evaluations or identification of a causal genetic mutation such as $A P P, P S E N 1$ or PSEN2. It was acknowledged that the genes that were linked by association, such as $A P O E$, were not sufficiently specific in this case (McKhann 2011).

\section{Possible Alzheimer's disease}

The criteria for possible Alzheimer's disease describe cases that present with an atypical course. These include sudden onset, no history of decline and absence of objective reported cognitive decline. This category would also include dementia of mixed aetiology.

Probable or possible Alzheimer's dementia with evidence of the Alzheimer's pathophysiological process

Those who are diagnosed as having probable Alzheimer's disease using the 1984 NINCDSADRDA criteria would still meet the proposed new criteria for probable Alzheimer's disease. However, those diagnosed with possible Alzheimer's disease using the 1984 criteria will need re-evaluation as they may not meet the new criteria thresholds.

The category of 'probable or possible Alzheimer's dementia with evidence of the Alzheimer's pathophysiological process' is intended for incorporation of biomarkers to increase the certainty that the basis of the clinical dementia syndrome is the Alzheimer's process. The NIAAlzheimer's Association workgroups currently do not recommend that biomarkers be used routinely. They report that the core clinical criteria should provide good diagnostic precision for the majority of patients. Biomarker testing is not yet widely available or standardised and the field as a whole requires further research. However, biomarkers have a role in research and as an option to provide more diagnostic certainty for the clinician.

This third category describes the group that meet the criteria for non-Alzheimer's dementia but have positive results in biomarkers or pathology at autopsy. Clinically, these patients would have been diagnosed with non-Alzheimer's dementia such as dementia with Lewy bodies but would meet biomarker or neuropathological criteria for Alzheimer's disease. This may include a group that have coexisting pathology. Pragmatic importance of this group is yet to be discovered.

\section{DSM-5}

The Neurocognitive Disorders Work Group of the American Psychiatric Association's DSM-5 Task Force has also been looking at their criteria in preparation for DSM-5. DSM-5 is scheduled for release in May 2013 (just as this article is going to press) and is in the final stages of development. The task force received public feedback to their proposed changes. The work group proposes the category 'neurocognitive disorder' (NCD) as it encompasses disorders of cognitive deficit that are particular to structural or metabolic brain disease. It believes that the term 'cognitive' has been used in various psychiatric and psychological settings covering all mental representations of informationprocessing and has therefore become too broad a term. In contrast, the term 'neurocognitive' has been used to describe links of cognitive function to areas of the brain or pathways in the brain (Ganguli 2011).

The work group introduced the concept of major $v$. minor NCD, where major refers to more severe disorders such as dementia. This appears to be related to individual levels of need and therefore, by definition, mild cognitive impairments would fall within the 'minor' NCD subgroup. Both levels of NCD then have subgroups that further describe aetiology, such as Alzheimer's disease subtype.

The work group does not propose removing the term dementia but intends to absorb it into the major NCD category. The group recognises that the term can be stigmatising in some diseases and age groups, and by including it in the major NCD category, the word can be used for subgroups when 
MCO answers

$1 \mathrm{c} 2 \mathrm{e} \quad 3 \mathrm{e} \quad 4 \mathrm{c} \quad 5 \mathrm{~b}$ appropriate. Thus, diagnoses such as dementia with Lewy bodies and Alzheimer's disease are likely to remain, whereas vascular dementia is subsumed within vascular cognitive impairment.

Interestingly, the work group recognises that functional impairment is a consequence of brain disease. It therefore suggests that functional impairment should not be used as a diagnostic criterion. Instead, it should be used to inform the severity of illness once a diagnosis has been made. This proposal would place greater emphasis on neuropsychological testing. Although this would lead to a more standardised, objective approach to diagnosis, there has been resistance to it because neuropsychological testing is not easily accessible and the decline is more difficult to measure. Consequently, a compromise has been proposed in which functional impairment that interferes with independence may be retained in some way in the diagnostic framework.

\section{Implications of changes for current and future clinical practice}

The introduction of the preclinical stage has highlighted the importance of emphasis on the early stages of disease. It is hoped that earlier diagnosis and treatment of Alzheimer's disease will lead to a better quality of life for patients by preserving their functions for longer. It should also enable cost savings for the health service as patients would require less intensive economic support in latter years (Getsios 2012).

It is becoming increasingly clear that a structured functional assessment will play a key role not only in identifying the level of impairment at initial assessment, but also in assessing the conversion from MCI to dementia. A variety of such assessment tools are being developed or validated for use in clinical practice (Gomar 2011). The use of combination of functional and cognitive scales appears to improve the sensitivity of diagnosis (Larner 2012). If biomarkers were also assessed clinicians could probably improve the diagnostic process further.

In view of the rate of conversion from MCI to dementia, recommendations have been made for monitoring for cognitive decline in people showing MCI so that treatment strategies can be initiated at the earliest point (Peterson 2001; Abert 2011). Clinical treatments in MCI are being investigated but no drugs have been licensed.

Although the NIA-Alzheimer's Association diagnostic criteria have not yet been put into practice, they have highlighted the following important ideas, of which clinicians should be aware.
- The pathological process of Alzheimer's disease is a long process.

- The emerging technology has allowed clinicians to define a preclinical stage. This could potentially increase treatment options, which may lead to secondary prevention of dementia in the future.

- Biomarkers can give more evidence at any stage of the disease and are particularly useful in diagnostically ambiguous cases.

- Clinical treatment at the stage of MCI is being investigated.

- The current emphasis is on early detection of Alzheimer's disease, but often the method of elucidating functional change is crude, possibly leading to an over-diagnosis of MCI rather than dementia. Use of formal functional assessments and even biomarkers can lead to a more robust diagnostic process in dementia.

\section{References}

Alagiakrishnan K, Gill SS, Fagarasanu, A (2012) Genetics and epigenetics of Alzheimer's disease. Postgraduate Medical Journal 88: 522-9.

Albert MS, DeKosky ST, Dickson D, et al (2011) The diagnosis of mild cognitive impairment due to Alzheimer's disease: recommendations from the National Institute on Aging-Alzheimer's Association workgroups on diagnostic guidelines for Alzheimer's disease. Alzheimer's \& Dementia 7: 270-9.

Bateman RJ, Xiong C, Benzinger TL, et al (2012) Clinical and biomarker changes in dominantly inherited Alzheimer's disease. New England Journal of Medicine 367: 795-804.

Doraiswamy PM (2012) Is the idle mind a Devil's workshop? Biological Psychiatry 71: 765-6.

Duara R, Loewenstein DA, Greig MT, et al (2011) Pre-MCI and MCl: neuropsychological, clinical, and imaging features and progression rates. American Journal of Geriatric Psychiatry 19: 951-60.

Dubois B, Albert ML (2004) Amnestic MCl or prodromal Alzheimer's disease? Lancet Neurology 3: 246-8.

Dubois B, Feldman HH, Jacova C, et al (2010) Revising the definition of Alzheimer's disease: a new lexicon. Lancet Neurology 9: 1118-27.

Fagan AM, Mintun MA, Mach RH, et al (2006) Inverse relation between in vivo amyloid imaging load and cerebrospinal fluid Abeta42 in humans. Annals of Neurology 59: 512-9.

Feldman HH, Jacova C (2005) Mild cognitive impairment. American Journal of Geriatric Psychiatry 13: 645-55.

Forsberg A, Engler H, Almkvist 0, et al (2008) PET imaging of amyloid deposition in patients with mild cognitive impairment. Neurobiology of Aging 29: 1456-65.

Ganguli M, Blacker D, Blazer DG, et al (2011) Classification of neurocognitive disorders in DSM-5: a work in progress. American Journal of Geriatric Psychiatry 19: 205-10.

Gauthier S, Reisberg B, Zaudig M, et al (2006) Mild cognitive impairment. Lancet 367: 1262-70.

Getsios D, Blume S, Ishak KJ, et al (2012) An economic evaluation of early assessment for Alzheimer's disease in the United Kingdom. Alzheimer's \& Dementia 8: 22-30.

Gomar JJ, Bobes-Bascaran MT, Conejero-Goldberg C, et al (2011) Utility of combinations of biomarkers, cognitive markers, and risk factors to predict conversion from mild cognitive impairment to Alzheimer disease in patients in the Alzheimer's disease neuroimaging initiative. Archives of General Psychiatry 68: 961-9.

Heinik J (2010) V. A. Kral and the origins of benign senescent forgetfulness and mild cognitive impairment. International Psychogeriatrics 22: 395-402. 
Jack CR Jr, Dickson DW, Parisi JE, et al (2002) Antemortem MRI findings correlate with hippocampal neuropathology in typical aging and dementia. Neurology 58: 750-7.

Jack CR Jr, Knopman DS, Jagust WJ, et al (2010) Hypothetical model of dynamic biomarkers of the Alzheimer's pathological cascade. Lancet Neurology 9: 119-28.

Jack CR Jr, Albert MS, Knopman DS, et al (2011) Introduction to the recommendations from the National Institute on Aging-Alzheimer's Association workgroups on diagnostic guidelines for Alzheimer's disease. Alzheimer's \& Dementia 7: 257-62.

Larner AJ, Hancock P (2012) Does combining cognitive and functional scales facilitate the diagnosis of dementia? International Journal of Geriatric Psychiatry 27: 547-8.

McKhann G, Drachman D, Folstein M, et al (1984) Clinical diagnosis of Alzheimer's disease: report of the NINCDS-ADRDA Work Group under the auspices of Department of Health and Human Services Task Force on Alzheimer's Disease. Neurology 34: 939-44.

McKhann GM, Knopman DS, Chertkow H, et al (2011) The diagnosis of dementia due to Alzheimer's disease: recommendations from the National Institute on Aging-Alzheimer's Association workgroups on diagnostic guidelines for Alzheimer's disease. Alzheimer's \& Dementia 7: 263-9.

National Institute for Health and Clinical Excellence (2012) Dementia: Supporting People with Dementia and Their Carers in Health and Social Care (NICE Clinical Guideline 42). NICE.
Petersen RC, Stevens JC, Ganguli M, et al (2001) Practice parameter. Early detection of dementia: mild cognitive impairment (an evidencebased review). Report of the Quality Standards Subcommittee of the American Academy of Neurology. Neurology 56: 1133-42.

Plassman BL, Langa KM, Fisher GG, et al (2008) Prevalence of cognitive impairment without dementia in the United States. Annals of Internal Medicine 148: 427-34

Sperling RA, Aisen PS, Beckett LA, et al (2011) Toward defining the preclinical stages of Alzheimer's disease: recommendations from the National Institute on Aging-Alzheimer's Association workgroups on diagnostic guidelines for Alzheimer's disease. Alzheimer's \& Dementia 7: $280-92$

Stephan BC, Matthews FE, Khaw KT, et al (2009) Beyond mild cognitive impairment: vascular cognitive impairment, no dementia (VCIND). Alzheimer's Research \& Therapy 1: 4

Strozyk D, Blennow K, White LR, et al (2003) CSF Abeta 42 levels correlate with amyloid neuropathology in a population-based autopsy study. Neurology 60: 652-6.

Valenzuela MJ, Matthews FE, Brayne C, et al (2012) Multiple biological pathways link cognitive lifestyle to protection from dementia. Biological Psychiatry 71: 783-91.

Walsh DM, Selkoe DJ (2007) A beta oligomers - a decade of discovery. Journal of Neurochemistry 101: 1172-84.

\section{MCOs}

Select the single best option for each question stem

\section{As regards biomarkers in Alzheimer's} disease:

a the first biomarker change in Alzheimer's disease is reflected by a decrease in CSF tau levels

b $\beta$ amyloidosis can only be detected in venous plasma samples

c amyloid- $\beta$ accumulation is not sufficient to cause disease progression

d PET imaging is estimated to be able to predict changes 25 years prior to symptoms

e all individuals that have positive biomarker results progress at the same rate.

\section{The DSM-5 Task force proposes:}

a that the term cognitive disorder be used to describe dementia

b removal of the term dementia from diagnostic nomenclature c that functional impairment should be used as a diagnostic criterion for Alzheimer's disease

d that neuropsychological testing would be unnecessary to diagnose dementia

e that $\mathrm{MCl}$ would be classified as minor neurocognitive disorder.

3 In preclinical Alzheimer's disease:

a there is no conceptual difference between brain reserve and cognitive reserve

b an active cognitive lifestyle does not protect against Alzheimer's disease

c patients show measurable changes on MMSE

$\mathrm{d}$ the pathological process of Alzheimer's disease generally takes 5 years

e preclinical staging will lead to cost savings for healthcare services.

4 Mild cognitive impairment (MCI):

a always leads to dementia

b of the non-amnestic form is associated with Alzheimer's disease c can often be differentiated from dementia by functional testing

d can only be caused by a neurological pathology

e has pharmacological treatment.

\section{As regards the new diagnostic criteria for} Alzheimer's disease:

a they exclude all other disease processes

b patients with a previous diagnosis of Alzheimer's disease would meet the new criteria for the disease

c biomarker technology should be used to support the diagnosis in all cases

$\mathrm{d}$ a diagnosis is impossible if there is no collateral information

e impairment in all domains of cognitive function should exist for a diagnosis. 